\title{
Research on Surface Micro Pattern Generation in Turn-Milling Considering Ball-End Milling Cutter Eccentricity
}

\author{
Huiqun Chen ${ }^{1,2, a}$, Jiliang $\mathrm{Hu}^{2, \mathrm{~b}}$ \\ ${ }^{1}$ School of Mechanical and Automotive Engineering, South China University of Technology, \\ Guangzhou 510640, China; \\ ${ }^{2}$ School of Mechanical and Electrical Engineering, Shenzhen Institute of Information Technology, \\ Shenzhen 518172, China; \\ achqleo@163.com, b chqleo@126.com
}

Keywords: turn-milling, surface micro-pattern, ball-end milling cutter, cutter eccentricity.

\begin{abstract}
The mathematical model of machined workpiece by orthogonal turn-milling with ball-end milling cutter is established, which takes the effect of ball-end milling cutter eccentricity into account. The simulation steps and flow chart of the algorithm are also given in this paper. The relation between representative turn-milling parameters (feed per tooth) and machined surface micro-pattern has been ascertained, which provides evaluation mechanisms reference for surface micro-pattern in the turnmilling affected by other machining parameters, the surface micro-pattern simulation is developed base on computer programming. The consistency of surface micro-pattern in simulation and machining experiment is verified, which is in the aspect of shapes and positions layout of peaks and valleys on the micro-pattern. This model is effective and feasible that it also can provide a basis for rational selection of cutting parameters in turn-milling.
\end{abstract}

\section{Introduction}

Turn-milling technology is an advanced machining method developed in 1980s[1,2].Instead of turning and milling, turn-milling can effectively utilize the existing cutters to process the workpieces of various materials, and realize the machining of various kinds of rotary parts and thin wall parts[3].The utility model has the advantages of high processing efficiency, high machining precision, long tool life, stable cutting, no chip breaking, and can finish the processing which is usually difficult to be realized by a single turning or a milling machine[4-6].

The machined surface quality is an important part of the metal cutting theory. As the pioneer of turn-milling technology, Schulz H and Spur G [7] from the Institute of Machine Tools and Production Engineering of Darmstadt University, carried out the roller bearing test by turn-milling, the surface integrity, geometric accuracy and chip formation of orthogonal and axial turn-milling were mainly studied. In recent years, some scholars in other countries have studied the surface quality of turnmilling. S.K.Choudhury and J.B.Bajpai[8] conducted the experiments for orthogonal turn-milling of mild-steel work piece with high speed steel milling cutters using planning of experiments technique to study the surface finish achieved. K. Arun Vikram and Ch. Ratnam[9] focused on effect of machining parameters on Surface Roughness (Ra) and Tool Vibrations (VIB) in manufacturing operational methods like tangential and orthogonal turn-milling processes. Single cut machining on A-axis of CNC Vertical Milling centre using HSS and carbide end mill cutters were adopted.Vedat Savas and Cetin Ozay [10] presented an approach for optimization of cutting parameters at cylindrical workpieces leading to minimum surface roughness by using genetic algorithm in the tangential turnmilling process. Then they presented an approach for optimization of the effects of the cutting parameters including cutter speed, workpiece speed, axial feed rate, and depth of cut on the surface roughness in the machining of $100 \mathrm{Cr} 6$ steel with tangential turn-milling method by using genetic algorithm (GA) [11]. Tangential turning-milling method has been determined to have optimum effects of cutting parameters on the machining of $100 \mathrm{Cr} 6$ steel. Lida Zhu,et al.[12]proposed an experimental study on the 3D chip morphology properties during orthogonal turnmilling of Al6061-T6.The effects of cutting parameters on turn-milling chip length and thickness were examined and discussed.In paper 
[13], a new two-dimensional stability model for orthogonal turn-milling was established in which both variable cutting depth and crosscoupling of the structural mode are considered. The stability lobe diagram was obtained using the full-discretization method. Umut Karaguzel, et al. [14] defined the effect of the tool axis offset based upon geometrical relationships which were used to develop a mechanistic force model.

As turn-milling is different from traditional cutting mechanism and application advantages [15, 16], it is necessary to comprehend the surface roughness and its influence factors. In addition, during the cutting process, the common normal of the machined surface and cutter spherical surface passes through the center of the cutter spherical surface, then the phenomenon of interference over cutting is easy to monitor and cutting motion path is easy to control. In this paper, the experimental research on the cutting process of ball-end milling by orthogonal turn milling is carried out, and the mathematical model of its surface micro-pattern is established, the surface micro-pattern is simulated based on computer programming, which provides a basis for rational selection of cutting parameters in turn-milling.

\section{Mathematical Model for Orthogonal Turn-Milling by Ball-End Milling Cutter}

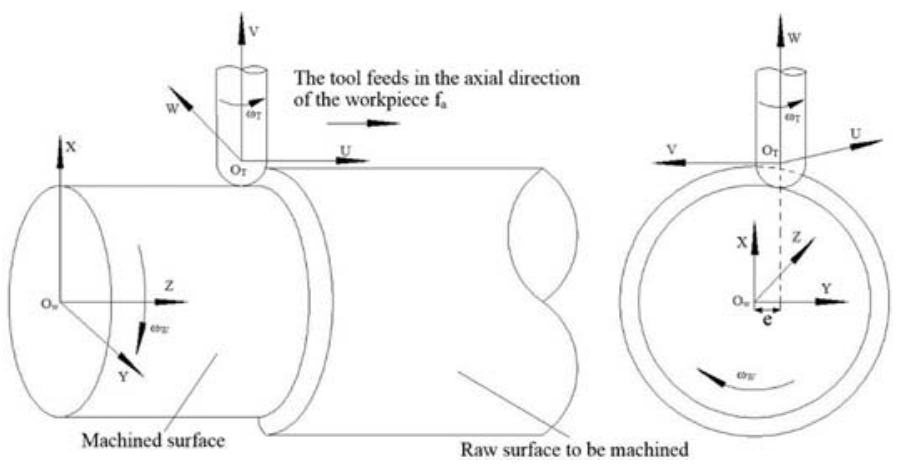

Fig.1 Mathematical model for orthogonal turn-milling by ball-end milling cutter

Fig. 1 shows the geometric mathematical model for orthogonal turn-milling cylinder by ball-end milling cutter.where $f_{a}$ is the tool feeds in the axial direction of the workpiece, e is the tool eccentricity, $\omega_{w}$ is the rotating angular speed of the workpiece, $\omega_{T}$ is the rotating angular speed of the workpiece. Supposing that the workpiece does not move and the tool moves relative to the workpiece. The derivation is as follows.

Firstly, the coordinate system $O_{W S 1}-X_{1} Y_{1} Z_{1}$ can be obtained by rotating $\omega_{W S} t$ angle around the $Z$ axis with the workpiece coordinate system $0_{W}-X Y Z$. The meaning of $S$ in $\omega_{W S}$ is orthogonal down milling, then the corresponding transformation matrix is $T_{1 S}$, which can be expressed as follows:

$$
T_{1 S}=\left[\begin{array}{cccc}
\cos \left(\omega_{W S} t\right) & -\sin \left(\omega_{W S} t\right) & 0 & 0 \\
\sin \left(\omega_{W S} t\right) & \cos \left(\omega_{W S} t\right) & 0 & 0 \\
0 & 0 & 1 & 0 \\
0 & 0 & 0 & 1
\end{array}\right]
$$

Secondly, the coordinate system $\mathrm{O}_{W S 2}-X_{2} Y_{2} Z_{2}$ can be obtained by rotating $\pi / 2$ angle around the $Y_{1}$ axis with the coordinate system $\mathrm{O}_{W S 1}-X_{1} Y_{1} Z_{1}$, then the corresponding transformation matrix is denoted as $T_{2 S}$. Finally, the coordinate system $O_{W S 3}-X_{3} Y_{3} Z_{3}$ can be obtained by rotating $\pi$ angle around the $Z_{2}$ axis with the coordinate system $\mathrm{O}_{W S 2}-X_{2} Y_{2} Z_{2}$, then the corresponding transformation matrix is denoted as $T_{3 S}$. After the above three steps transformation, each coordinate axis in coordinate system $\mathrm{O}_{W S 3}-X_{3} Y_{3} Z_{3}$ is consistent with the corresponding coordinate axis in tool coordinate system $0_{T}-U V W$, the total transformation matrix of the orthogonal down milling by the ball-end cutter is denoted as $\mathrm{T}_{S}$ and can be expressed as:

$$
\mathrm{T}_{S}=\mathrm{T}_{1 S} * \mathrm{~T}_{2 S} * \mathrm{~T}_{3 S}
$$

Compared with the orthogonal down milling, the mathematical model for the orthogonal up milling of ball-end milling cutter is only the opposite rotation direction of the workpiece, and the other processing conditions remain unchanged. Therefore, the workpiece coordinate system of ballend milling in orthogonal up milling $\mathrm{O}_{W}-X Y Z$, can be rotated $-\omega_{W N} t$ angle around the $\mathrm{Z}$ axis to 
obtain the coordinate system $\mathrm{O}_{W N 1}-X_{1} Y_{1} Z_{1}$, the meaning of $N$ in $\omega_{W N} t$ is orthogonal up milling. The corresponding transformation matrix $\mathrm{T}_{1 N}$ can be represented in Eq. (3).

$$
T_{1 N}=\left[\begin{array}{cccc}
\cos \left(\omega_{W N} t\right) & \sin \left(\omega_{W N} t\right) & 0 & 0 \\
-\sin \left(\omega_{W N} t\right) & \cos \left(\omega_{W N} t\right) & 0 & 0 \\
0 & 0 & 1 & 0 \\
0 & 0 & 0 & 1
\end{array}\right]
$$

Secondly, after two coordinate changes, the coordinate system $\mathrm{O}_{W N 2}-X_{2} Y_{2} Z_{2}$ can be obtained by rotating $\pi / 2$ angle around the $Y_{1}$ axis with the coordinate system $O_{W N 1}-X_{1} Y_{1} Z_{1}$, and the corresponding transformation matrix is denoted as $T_{2 N}$. Finally, the coordinate system $\mathrm{O}_{W N 3}-$ $X_{3} Y_{3} Z_{3}$ can be obtained by rotating $\pi$ angle around the $Z_{2}$ axis with the coordinate system $\mathrm{O}_{W N 2}-$ $X_{2} Y_{2} Z_{2}$, and the corresponding transformation matrix is denoted as $T_{3 N}$. After the above three steps transformation, each coordinate axis in coordinate system $O_{W N 3}-X_{3} Y_{3} Z_{3}$ is consistent with the corresponding coordinate axis in tool coordinate system $\mathrm{O}_{T}-U V W$, the total transformation matrix of the orthogonal up milling by the ball-end cutter is denoted as $\mathrm{T}_{N}$ and can be expressed as:

$$
\mathrm{T}_{N}=\mathrm{T}_{1 N} * \mathrm{~T}_{2 N} * \mathrm{~T}_{3 N}
$$

Therefore, the mathematical model for orthogonal down milling of ball-end milling cutter is represented by Eq. (2), while the mathematical model for orthogonal up milling is represented by Eq. (4).

The coordinate origin of tool coordinate system $\mathrm{O}_{T}-U V W$ in the coordinate system $\mathrm{O}_{W 3}-$ $X_{3} Y_{3} Z_{3}$ are expressed as follows:

$$
\left\{\begin{array}{l}
x_{O T}^{\prime}=f \cdot t \\
y_{O T}^{\prime}=e \\
z_{O T}^{\prime}=R+R_{w}-a_{p}
\end{array}\right.
$$

Where $f$ denotes the tool feed along the workpiece axial, $e$ is the tool eccentricity, $\mathrm{R}$ is the tool radius, $R_{W}$ is the workpiece radius before processing, $a_{p}$ is the cutting depth,and $t$ is the time experienced from the start of the process to the current moment.

The kinematic error of machine spindle can be taken into account in the simulation that allows the simulation results to be closer to reality. The kinematic error of machine spindle mainly includes rotational eccentricity and the axial movement of the rotating spindle. First of all, supposing that the radius of the eccentric circle of the rotating spindle is $\Delta D_{1} / 2$, and the axial drift amplitude is $\Delta \mathrm{D}_{2} / 2$. Then considering the vibration error of the tool during the machining process, the transformation matrix of ball-end milling cutter center of orthogonal down milling or up milling in the $\mathrm{O}_{W 3}-X_{3} Y_{3} Z_{3}$ coordinate system can be expressed[17]:

$$
T_{Q}=\left[\begin{array}{cccc}
1 & 0 & 0 & f \cdot t+\Delta \mathrm{D}_{1} \cdot \frac{\cos \left(\Delta \alpha_{1}+\varphi_{i, 1}-\omega_{T} \cdot t\right)}{2} \\
0 & 1 & 0 & \Delta \mathrm{D}_{1} \cdot \frac{\sin \left(\Delta \alpha_{1}+\varphi_{i, 1}-\omega_{T} \cdot t\right)}{2} \\
0 & 0 & 1 & R+R_{w}-a_{p}+\Delta \mathrm{D}_{2} \cdot \frac{\sin \left(\Delta \alpha_{2}+\varphi_{i, 1}-\omega_{T} \cdot t\right)}{2} \\
0 & 0 & 0 & 1
\end{array}\right]
$$

Where $\Delta \alpha_{1}, \Delta \alpha_{2}$ are the initial phase angle of spindle rotational eccentricity and axial movement, respectively, $\varphi_{i, 1}$ is initial phase angle of tool, and $\omega_{T}$ is the angular speed of tool spindle.

The transformation matrix of tool center axis offset relative to workpiece centerline is denoted as $_{e}$, which can be expressed as:

$$
T_{e}=\left[\begin{array}{llll}
1 & 0 & 0 & 0 \\
0 & 1 & 0 & e \\
0 & 0 & 1 & 0 \\
0 & 0 & 0 & 1
\end{array}\right]
$$

In summary, when the ball-end milling cutter has a change of position in the turn-milling process and is affected by many factors, the coordinates of any point $\mathrm{P}$ on ball-end cutter edge in the workpiece coordinate system $\mathrm{O}_{W}-X Y Z$ can be derived as follow: 


$$
\left[\begin{array}{l}
x \\
y \\
x \\
1
\end{array}\right]=T_{Q} \cdot T_{e} \cdot T_{x} \cdot T_{y} \cdot\left[\begin{array}{c}
u \\
v \\
w \\
1
\end{array}\right]
$$

Where $(\mathrm{x}, \mathrm{y}, \mathrm{z})$ are the coordinates of any point on ball-end cutter edge in the workpiece coordinate system, $(\mathrm{u}, \mathrm{v}, \mathrm{w})$ are coordinates of any point on ball-end cutter edge in the tool coordinate system, $T_{x}$ is the tool spindle tilt angle conversion matrix when milling mode is $x$ (There are four types of milling: push milling $T$, pull milling $\mathrm{L}$, down milling $\mathrm{S}$ and up milling $\mathrm{N}$ ). $T_{y}$ is the end blade type ( $y=1$ represents the standard form, $y=2$ represents the flat form, $y=3$ represents $V$-shaped form, and $\mathrm{y}=4$ represents $\mathrm{U}$-shaped form).

\section{Simulation Steps and Flow Chart of Algorithm}

Turn-milling compound includes milling cutter rotation, workpiece rotation, milling cutter feeding and moving axially along the workpiece $[18,19]$.

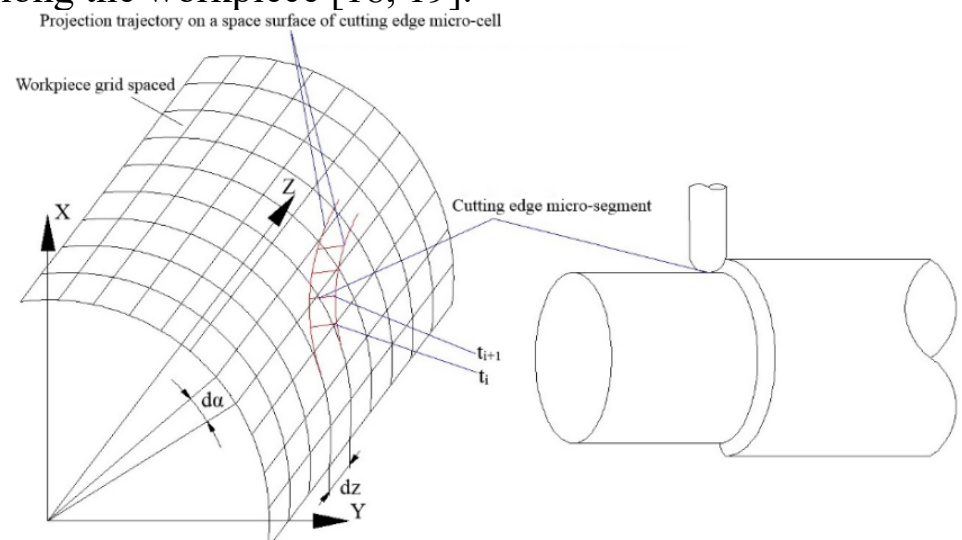

Fig. 2 Grid on workpieces surface

Owing to the main object of orthogonal turn-milling is large revolving shaft and slender shaft parts, so the linear feed motion of the milling cutter is generally axial feed, and seldom using the circumferential feed [20]. Therefore, this article mainly discusses three kinds of movement: the milling cutter rotation, the workpiece rotation, the milling cutter along the workpiece axial feed. In the process of turn-milling, the moving track of the tool relative to the workpiece is a continuous spiral. The 3D mesh model is adopted in the simulation system. The cylindrical workpiece is divided into the grid in axial and circumferential directions (as shown in Fig.2). In order to analyze the micro pattern of the machined surface, it is necessary to discretize the machining surface, the cutter edge and the machining time. By calculating the coordinates of the points in the workpiece coordinate at different times, whether the cutter has been cut into the workpiece by comparing these coordinates and the corresponding coordinates on the machined surface. According to the residual height of the machined surface, the micro-pattern of the milling surface can be obtained.

The micro-pattern simulation steps in turn-milling are as follows:

(1) The workpiece is divided into $m$ and $n$ respectively in axial feed direction ( $\mathrm{Z}$ direction) and circumferential feed direction. The spaces are $\mathrm{dz}$ and $\mathrm{d} \alpha$ ( $\alpha$ is the central angle in the circumferential direction), the workpiece surface is divided into $\mathrm{m} \times \mathrm{n}$ grids, the $\mathrm{m} \times \mathrm{n}$ grid-points matrix $\mathrm{H}[\mathrm{i}, \mathrm{j}](\mathrm{i}=$ $1,2, \cdots \mathrm{m}, \mathrm{j}=1,2, \cdots \mathrm{n}$ ) represents the radial heights corresponding to the position points on workpiece surface. Before turn-milling, the initial values of the matrix elements are $\mathrm{R}_{W}$ (workpiece radius). 


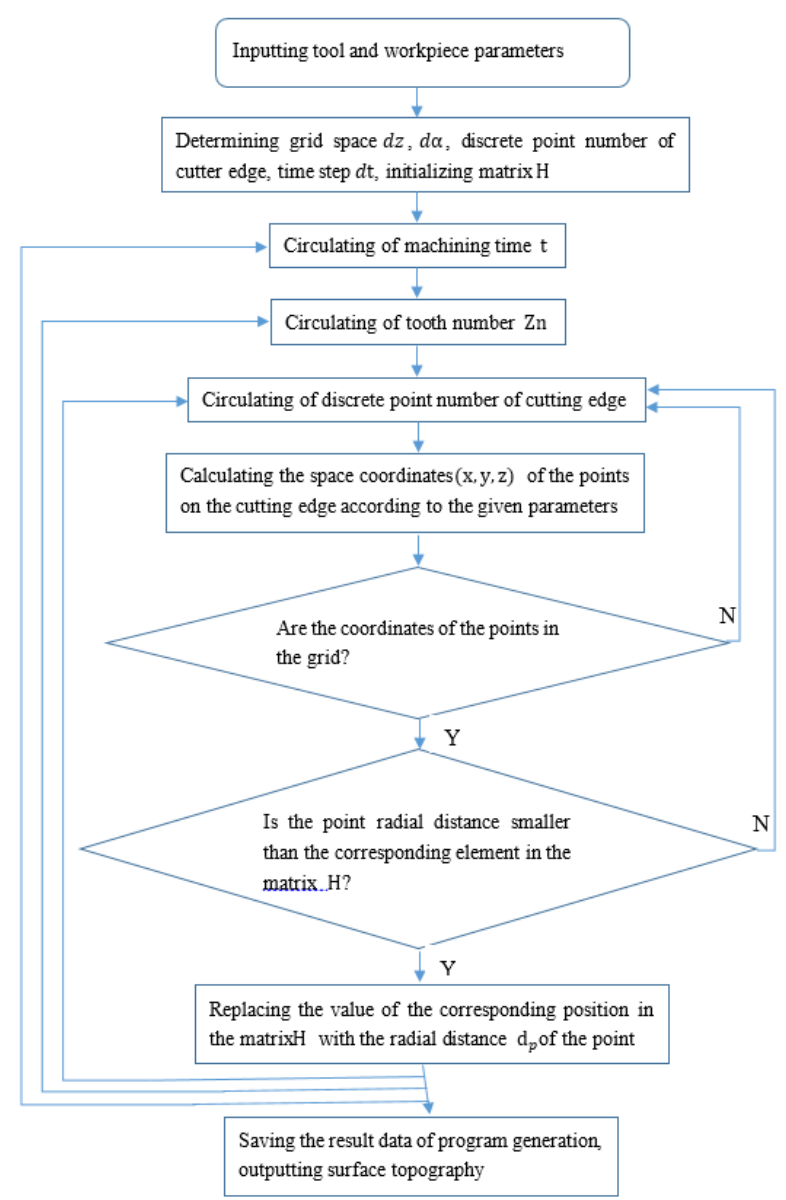

Fig.3 Flow chart of surface topography simulation algorithm

(2) The cutting edge and processing time of the tool are discretized. Firstly, according to the calculation model mentioned above, the coordinates of the points on the cutter edge in the workpiece coordinate system are obtained, then these coordinates are compared with the corresponding point coordinates on the machining surface, and then the residual heights of the workpiece surface are determined.

(3) In the simulation process, each residual height is continuously replaced by the the radial length $\mathrm{d}_{p}$ of point $P$. Firstly, the radial length of point $P$ on the cutter edge is calculated, the radial length of the point $P$ is compared with the height value $\mathrm{H}[\mathrm{i}, \mathrm{j}]$ of the corresponding grid point preserved in the previous turn-milling, Whend $\mathrm{d}_{p}<\mathrm{H}[\mathrm{i}, \mathrm{j}]$, which indicates that point $P$ on the cutter edge has been cut into the workpiece, then the corresponding element in the matrix $\mathrm{H}$ is modified by current $\mathrm{d}_{p}$ of point $P$.

(4) The micro-pattern of the machined surface is drawn according to the matrix H. The flow chart of surface micro-pattern simulation algorithm is shown in Fig.3. This software can describe the surface micro-pattern of turn-milling under different processing conditions. Meanwhile, through this simulation software, we can analyze the influence of cutting parameters on the surface micro-pattern and determine the reasonable cutting parameters.

\section{Machining Experiment of Orthogonal Turn-Milling By Ball-End Milling Cutter}

The turn-milling experimental equipment was KVC650M vertical machining center made in China, the maximum speed of the tool was $8000 \mathrm{r} / \mathrm{min}$. CNC system was FANUC Series oi Mate-MODEL D of Japan, The compound machining of orthogonal turn-milling can be realized on this machine tool. The workpiece material is YL12-CZ aluminum bar, the micro-pattern of the machined surface is observed by binocular optical microscope. 


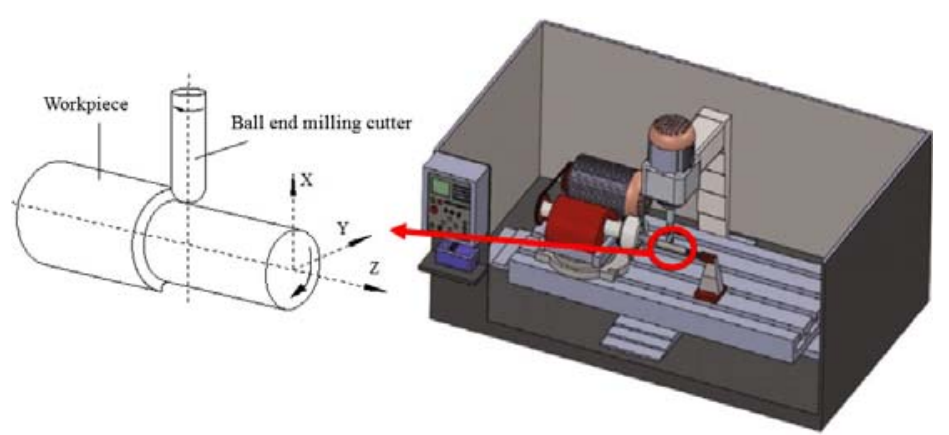

Fig.4 Schematic diagram of the experimental setup

\subsection{Experiment Scheme.}

The milling parameters of the ball-end milling cutter are given in Table 1. The microscopic pattern of the surface can be observed with 30 times microscope and taken pictures. The surface pattern of actual machining and simulation result are compared under the same turn-milling parameters, so as to verify the feasibility of the simulation system of surface micro-pattern, schematic diagram of the experimental setup is shown in Fig.4.

Table 1 A set of cutting parameters.

\begin{tabular}{ccccccccc}
\hline $\begin{array}{c}\text { Tool } \\
\text { radius } \\
(\mathrm{mm})\end{array}$ & $\begin{array}{c}\text { Workpiece } \\
\text { radius }(\mathrm{mm})\end{array}$ & $\begin{array}{c}\text { Axial feed } \\
(\mathrm{mm} / \mathrm{min})\end{array}$ & $\begin{array}{c}\text { Tool speed } \\
(\mathrm{r} / \mathrm{min})\end{array}$ & $\begin{array}{c}\text { Workpiece } \\
\text { speed }(\mathrm{r} / \mathrm{min})\end{array}$ & $\begin{array}{c}\text { Cutter tooth } \\
\text { number }\end{array}$ & $\begin{array}{c}\text { Cutting } \\
\text { depth(mm) }\end{array}$ & $\begin{array}{c}\text { Eccentricity } \\
(\mathrm{mm})\end{array}$ & $\begin{array}{c}\text { helix angle } \\
(\mathrm{radian})\end{array}$ \\
\hline 1.5 & 3 & 3 & 1000 & 40 & 2 & 0.5 & 0.3 & $\pi / 6$ \\
\hline
\end{tabular}

\subsection{Comparison and Analysis of Experimental and Simulation Results.}

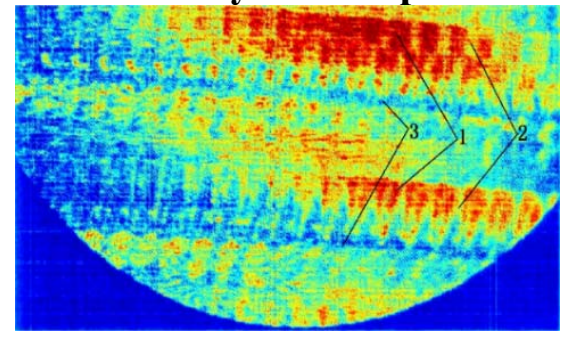

(a)

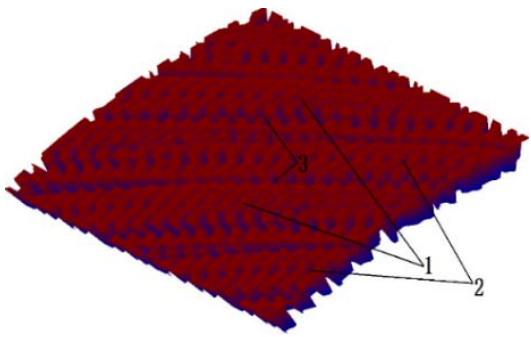

(b)

Fig.5 Surface micro-pattern of measured result (a) and simulated result (b)

Fig.5 (a) shows the microscopic pattern of the actual machined surface taken from the microscope, and Fig.5 (b) shows the surface micro-pattern simulated under the same turn-milling parameters. Comparison between simulated results and measured ones is conducted in the aspect of shapes and positions layout of peaks and valleys on the micro-pattern, in Fig.5, mark1 is raised peaks, mark2 is sunken valleys, and mark3 is permutation boundaries. As can be seen from Fig.5, the simulation results are in good agreement with the actual observation. Because it is cylindrical surface, the microscope can only observe the local part clearly, therefore, the topography observed on the right side of Fig.5 (a) is slightly blurred.

\section{Influence Analysis of Different Axial Feed on Surface Micro Topography}

Table 1 is the machining parameters of the outer cylindrical surface with axial feed $\mathrm{f}=$ $3 \mathrm{~mm} / \mathrm{min}$, and the other machining parameters of axial feedf $=5 \mathrm{~mm} / \mathrm{minand} \mathrm{f}=12 \mathrm{~mm} / \mathrm{min}$ are the same as table 1, Fig. 6 shows the micro topography simulation of ball-end milling cutter under different axial feeds. 


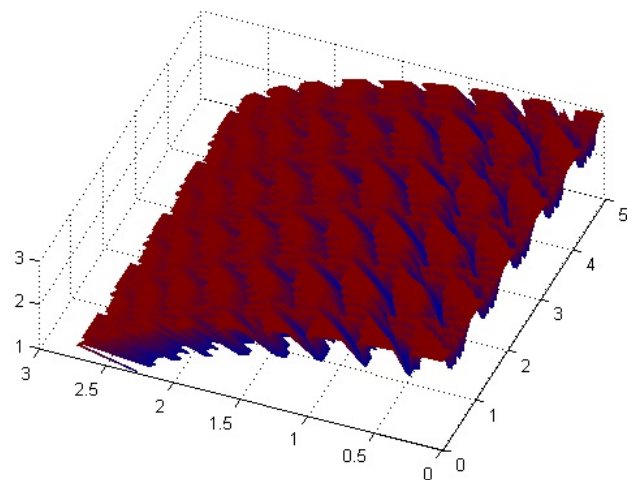

(a)

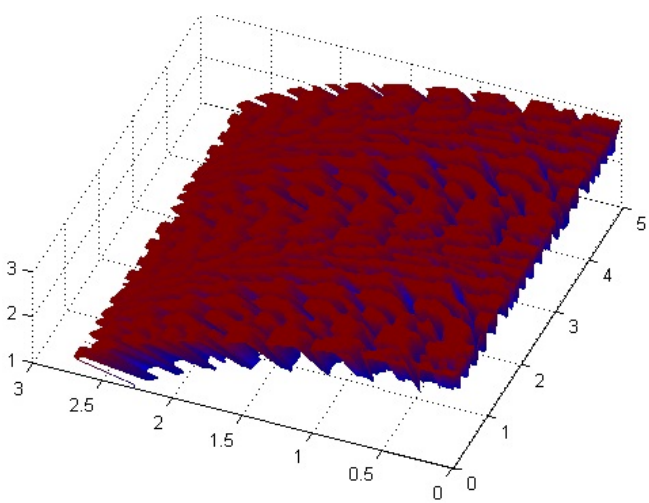

(b)

Fig.6 Surface micro topography simulation of axial feed $\mathrm{f}=5 \mathrm{~mm} / \mathrm{min}$ (a) and $\mathrm{f}=$ $12 \mathrm{~mm} / \mathrm{min}(\mathrm{b})$

By comparing Fig.5 (a) and Fig.5 (b), it can be seen that the row spacing of Fig.5 (b) is obviously larger than Fig.5 (a),and the scallop height of surface topography in Fig.5 (b) is the higher than that in Fig.5(a). That is to say, the surface roughness of Fig. 5 (b) is larger than that of Fig. 5 (a).Therefore, on the basis of guaranteed efficiency, the axial feed value should be as small as possible in actual processing, so that the surface roughness can be reduced.

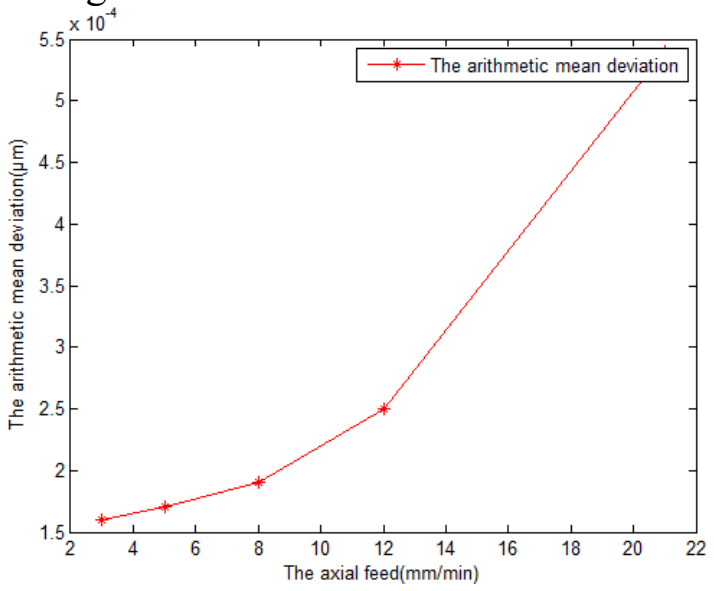

Fig.7 Change curve of arithmetic mean deviation with axial feed

Fig. 7 is the arithmetic mean deviation of the micro geometry topography machined by ball-end milling cutter, which varies with the axial feed. As can be seen from the figure, the arithmetic mean deviation shows an increasing trend with the increase of axial feed in a certain range. The arithmetic average deviation increases slowly between the axial feed $2 \mathrm{~mm} / \mathrm{min}$ and $13 \mathrm{~mm} / \mathrm{min}$, but after the axial feed $13 \mathrm{~mm} / \mathrm{min}$,it increases sharply, Which shows that if the axial feed is large, the effect of the axial feed on the surface roughness is relatively large, and it has a certain guiding significance for the actual processing.

\section{Summary}

In this paper, the orthogonal test method of ball-end milling cutter and cylindrical workpiece has been adopted, the mathematical model of the surface micro-pattern of ball-end milling cutter is established, which takes the effect of ball-end milling cutter eccentricity into account. The simulation software of surface micro-pattern is developed, and then computer simulation is done. The surface micro-pattern is simulated under different cutting parameters, and compared with actual cutting experiment in the aspect of shapes and positions layout of peaks and valleys on the micro-pattern, which shows that the mathematical model established in this paper can predict the surface microtexture of orthogonal turn-milling with higher reliability. In this paper, the influence of different axial feed on the micro topography of the machined surface is studied, the influence of the axial feed rate on the surface topography and the 3D surface roughness is relatively large, when the axial feed is in a certain range, the arithmetic mean deviation changes slightly. 


\section{Acknowledgements}

The authors gratefully acknowledge the financial supports of Guangdong Natural Science Foundation of China (Grant no.2015A030313590), Guangdong science and technology innovation project (Grant no.pdjhb0863), the Program for Scientific and Technological Innovation in Shenzhen of China (Grant nos.JCYJ20150417094158028, JCYJ20160415113818087), and Guangdong Science Planned Project of China (Grant no.2012JK291).

\section{References}

[1]. Lida Zhu,Haonan Li, Weili Liang,et al. A web-based virtual CNC turn-milling system.Int J Adv Manuf Technol.Vol.78(2015) No.(1-4),p. 99-113.

[2]. Lida Zhu, Zenghui Jiang,Jiashun Shi,et al.An overview of turn-milling technology.Int J Adv Manuf Technol.Vol.81(2015) No.(1-4),p.493-505.

[3]. Vedat Savas,Cetin Ozay. Analysis of the surface roughness of tangential turn-milling for machining with end milling cutter.Journal of Materials Processing Technology.Vol.186(2007) No. (1-3), p.279-283.

[4]. Umut Karagüzel,Emre Uysal,Erhan Budak,et al. Analytical modeling of turn-milling process geometry, kinematics and mechanics. International Journal of Machine Tools and Manufacture, Vol.91(2015) p.24-33.

[5]. Wenwang Qiu,Qiang Liu,Ji Ding,et al. Cutting force prediction in orthogonal turn-milling by directly using engagement boundaries.Int J Adv Manuf Techno.(2015) No.(1), p.1-13.

[6]. Wenwang Qiu,Qiang Liu,Songmei Yuan,et al. Modeling of cutting forces in orthogonal turnmilling with round insert cutters. Int J Adv Manuf Technol. Vol.78(2015) No.(5-8), p.1211-1222.

[7]. [7]Schulz H,Spur G.High speed turn-milling-a new precision manufacturing technology for the machining of rotationally symmetrical workpieces.CIRP Annals-Manufacturing Technology. Vol.39(1990) No.(1), p.107-109.

[8]. S.K.Choudhury, J.B.Bajpai. Investigation in orthogonal turn-milling towards better surface finish.Journal of Materials Processing Technology. Vol.170(2005) p.487-493.

[9]. K.Arun Vikrama,Ch.Ratnamb,K.Sankar Narayana.Vibration diagnosis and prognostics of Turnmilling operations using HSS and carbide end mill cutters. Procedia Technology. Vol.23(2016) p.217-224.

[10]. Vedat Savas,Cetin Ozay. The optimization of the surface roughness in the process of tangential turn-milling using genetic algorithm.Int J Adv Manuf Technol. Vol.37(2008) p.335340.

[11]. Vedat Savas,Cetin Ozay,Hasan Ballikaya.Experimental investigation of cutting parameters in machining of $100 \mathrm{Cr} 6$ with tangential turn-milling method.Adv. Manuf..Vol.4(2016) No.(1),p.97104.

[12]. Lida Zhu,Xiaoliang Jin,Changfu Liu. Experimental investigation on 3D chip morphology properties of rotary surface during orthogonal turn-milling of aluminum alloy.Int J Adv Manuf Technol.Vol.84(2016) No.(5-8), p.1253-1268.

[13]. Rong Yan,Xiaowei Tang,F. Y. Peng,et al. The effect of variable cutting depth and thickness on milling stability for orthogonal turn-milling.Int J Adv Manuf Technol. Vol.82(2007) No.(14),p.765-777.

[14]. Umut Karaguzel,Emre Uysal,Erhan Budak,et al. Effects of tool axis offset in turn-milling process.Journal of Materials Processing Technology. Vol.231(2016) p.239-247. 
[15]. E Uysal,U Karaguzel,E Budak,et al.Investigating Eccentricity Effects in Turn-milling Operations.Procedia CIRP. Vol.14(2014) No.(14), p.176-181.

[16]. E Kai,T Furukawa,K Yamaguchi,et al.Microcutting using a micro turn-milling machine.Precision Engineering.Vol.44(2016) p.81-86.

[17]. ZH Jiang,HY Pan.Movement modeling and simulation on machining equidistant profile by orthogonal turn-milling.Journal of Harbin Institute of Technology. Vol.41(2009) No. (12), p.259262.

[18]. Cai Yonglin, Ma Yemeng, Huang Chao.Swept area modeling and cutter wear study in turnmilling. Int J Adv Manuf Technol.Vol.80(2015) No.(5-8),p.775-789.

[19]. S.K. Choudhury,J.B. Bajpai.Investigation in orthogonal turn-milling towards better surface finish.Journal of Materials Processing Technology. Vol.170(2005) No.(3),p.487-493.

[20]. [20]JMC Filho.Prediction of cutting forces in mill turning through process simulation using a five-axis machining center.Int J Adv Manuf Technol.Vol.58(2012) No.(1-4), p.71-80. 